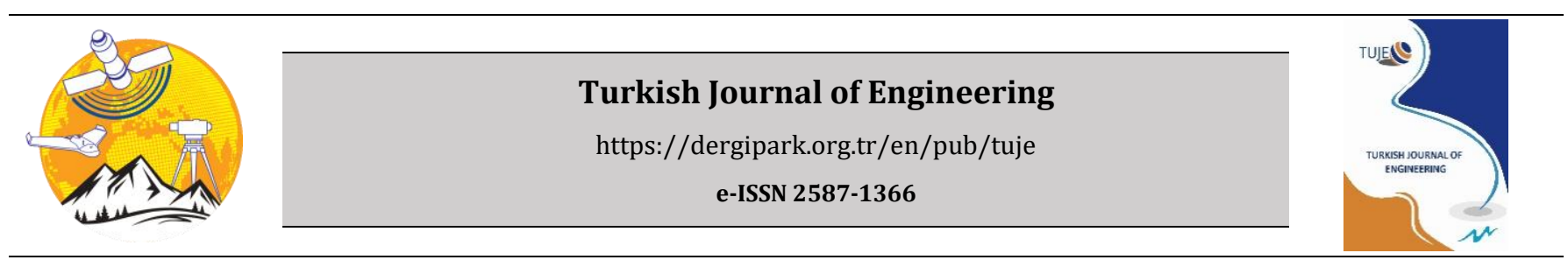

\title{
Slow-light effect in symmetry-reduced non-defect photonic crystals
}

\author{
Ibrahim Halil Giden* (D) \\ ASELSAN Academy, Ankara, Turkey
}

\author{
Keywords \\ Integrated circuits \\ Photonic crystals \\ Slow light \\ Light-matter interaction \\ Mode-order conversion
}

\begin{abstract}
In this study, a two-dimensional low-symmetric photonic crystal (PC) configuration with elliptical geometry is presented and its slow-light (SL) effect is investigated. Reducing the symmetry in the PC unit cell provides slow modes at the higher transverse electric bands. The calculated group index and the corresponding normalized bandwidth equal to $\left\{\mathrm{n}_{\mathrm{g}}\right.$, $B W\}=\{63.56,0.0065\}$. That corresponds to a value of figure of merit $(F O M)=0.4344$ defined by the product of the average group index and the normalized bandwidth, FOM $=\left\langle n_{g}\right\rangle B W$, which is comparable to the values available in literature. Tracing the whole edges of the Brillouin zone, strongly excited SL modes are observed only along $\Gamma-\mathrm{X}$ but not along $\Gamma-\mathrm{X}^{\prime}$ or $\Gamma-\mathrm{M}$. That condition allows for the design of low-symmetric PC waveguides with finite thicknesses at the expense of lowering group index value. The SL effect is still obtained for the proposed lowsymmetric PCs having finite thicknesses, which is numerically proved via finite-difference time-domain methods. It is important to note that non-dispersive SL Bloch modes exist through the non-zero $k$-vector components of Brillouin zone. Hence, such a defect-free (without either point- or line- defect) SL PC design may have a great potential for the use of compact photonic devices such as in optical switching and biochemical sensing applications.
\end{abstract}

\section{INTRODUCTION AND DISPERSION RELATIONS OF PROPOSED ELLIPTIC PHOTONIC CRYSTALS}

Photonic Crystals (PCs) are periodically modulated artificial materials that enable controlling the motion of light propagation as well as manipulating the photon velocity (Joannopoulos et al. 2008). Slowing down the flow of light enhances the light-matter interaction in a material. Photonic devices based on slow-light (SL) waveguides can be considered as key elements in optical communication systems to address bandwidth and switching power requests. Such devices also support enhanced nonlinearities, enabling the realization of compact low power all-optical switching devices (Khurgin \& Tucker, 2018). Group velocity reduction is achieved near the band-gap edges and slow-light regimes exist at the flattened bands. However, slow modes near the band edges are observed to be highly dispersive and very lossy, which limits its use for efficient SL applications (Engelen et al. 2006). For that reason, different methods such as structural modifications of the photonics waveguide (Üstün \& Kurt 2010; Üstün \& Kurt 2012), variation of material type (Wu et al. 2013) or implementation of coupled waveguide systems (Mori and Baba, 2005) have been applied to engineer the dispersion curves of the defect modes. Nevertheless, such defect-based SL waveguides may expose to very high extrinsic losses (Krauss, 2007) due to the structural imperfections. Apart from these approaches, researchers have continued to perform studies that explore basic photonic crystal waveguide (PCW) structures. PCWs subject to various different modifications were studied to enhance slow wave performance of propagating light pulses (Moghaddam \& Fleury, 2019; Hocini et al. 2018; Schulz et al. 2017; Han et al. 2015; Varmazyari et al. 2014; Khodamohammadi et al. 2015).

Slotted PCW configurations are suitable photonic structures to obtain slow light thanks to the availability for infiltration of slot region with low index material such as polymers and liquids/gases. As a result, slow light parameters can be externally tuned and such tuning capability could be implemented for efficient sensing applications (Kassa-Baghdouche \& Cassan, 2018; KassaBaghdouche \& Cassan, 2019; Zhao et al. 2014). Different optimization methods that target slow light studies considering group velocity dispersion, bandwidth, 

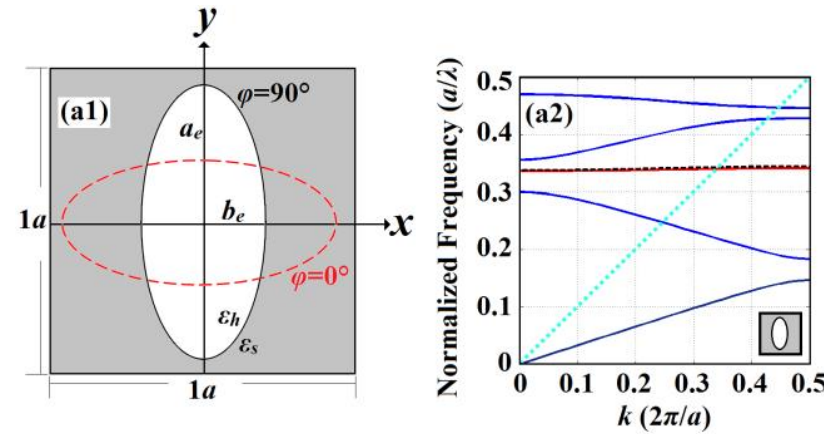
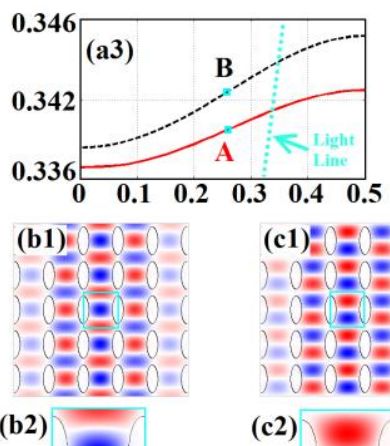
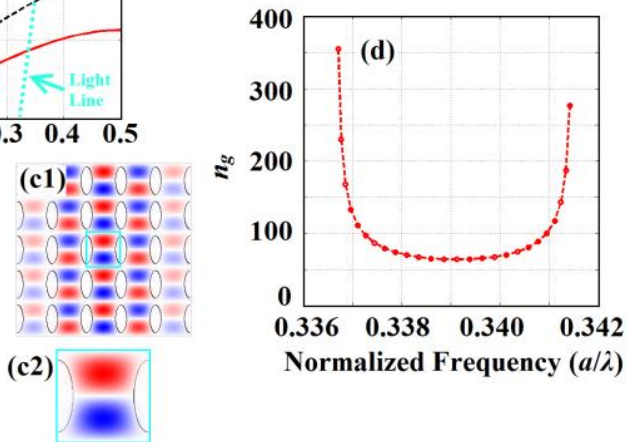

Figure 1. (a1) Schematic view of square lattice elliptic hole PC unit cell with its geometrical parameters. (a2) TE mode band structure of square-lattice elliptic PC holes with the dimensions of $\left(a_{e}, b_{e}, \varphi\right)=\left(0.40 a, 0.16 a, 90^{\circ}\right)$ and (a3) its close inspection at third TE band. The corresponding magnetic mode profiles of degenerate modes (b1) A and (c1) B, and their zoomed views at unit-cell scale: (b2) for the even mode, A, and (c2) for the odd mode, B. (d) The group index spectrum of the corresponding mode, A. Regarding mode profiles are calculated at the $k$-points signed with square markers in (a3).

normalized delay-bandwidth product and fabrication issues have been summarized in Zhao et al. (2015). It was indicated in Bagci \& Akaoglu (2015) that performance of optical devices such as buffering and electro-optic modulation could be improved via slow light concept. An optimization algorithm was implemented in Mirjalili \& Mirjalili (2014) and Mirjalili (2014) to obtain PCW with oval shaped holes and to enhance high normalized delaybandwidth product parameter.

Symmetry reduction in PC unit cell, i.e., breaking the rotational symmetry of PCs, facilitates the realization of peculiar optical characteristics such as complete photonic band gaps (Giden \& Kurt 2012), super collimation (Gumus et al. 2018) and graded refractiveindex modulation (Giden et al. 2015). The present study deals with SL in a low-symmetric PC waveguide that does not include either line- or point defects, which is termed as "defect-free" in the rest of the study. A twodimensional (2D) PC structure is considered, which is formed by square lattice elliptical holes and the PC unitcell geometry is schematically shown in Fig. 1(a1). The holes are assumed to be hollow, $\varepsilon_{\mathrm{h}}=1$ and the slab is made of $\mathrm{Si}-, \varepsilon_{s}=(3.45)^{2}$. The rotation angle, the major and minor radii of vertically-oriented elliptic $\mathrm{PC}$ holes are set to be $\left(a_{e}, b_{e}, \varphi\right)=\left(0.40 a, 0.16 a, 90^{\circ}\right)$ so that the regarding eccentricity equals $e=b_{\mathrm{e}} / a_{\mathrm{e}}=0.40$. The lattice constant is fixed at 1.0 a. It's well-known that reducing the symmetry of primitive PC cell influences the dispersion properties of the PC modes especially at high symmetry points (Trifonov et al. 2004; Giden et al. 2014). For that reason, two-fold (C2) rotationally symmetric PC unit cell with elliptical hole is chosen rather than regular one with circular hole in order to trigger the slow light effect.

Throughout the study, the polarization of light is transverse electric (TE), i.e., the magnetic field is perpendicular to the plane of propagation. Dimensions of the PC waveguide are arranged based on the future applications such as SL and mode-order conversion. Band structure as well as group index calculations are carried out using plane wave expansion method (Johnson and Joannopoulos 2001). 2D finite-difference time-domain (FDTD) verifications are also performed (Oskooi et al. 2010). In all numerical calculations, only TE polarization is employed and the FDTD grid size is set to $\Delta x=\Delta y=a / 30$. The computational domain is surrounded by perfectly-matched layers (PMLs) to remove undesired back reflections at the boundaries. Plane wave is incident to the designed medium for steady-state field calculations.

For information carrying optical pulse, the velocity of its envelope is measured as a group velocity, $v_{g}$. The term "slow light" (SL) implies the group velocity of the wave packet to be smaller than the speed of light in vacuum, $c$. Group index is, then, described by the following relation: $n_{g}=c \cdot\left(\frac{\partial \omega}{\partial k}\right)^{-1}=c / v_{g}$, where $\omega$ and $k$ denote the radial frequency and wavevector, respectively. Figure 1(a2) represents the dispersion diagram of designed 2D square lattice PC unit cell with elliptic holes (given as an inset in the figure) for TE modes in $\Gamma-\mathrm{X}$ direction. Dashed lines in the figure imply the light-line. Degenerate modes occur at the third TE band, which is surrounded by a box in Fig. $1(\mathrm{a} 2)$, and close-up view of the degenerate modes is shown in Fig. 1(a3). The magnetic field distributions of degenerate modes A and B are represented in Figs. 1(b1) and 1(c1), respectively. Unlike SL Bloch modes existing at the high symmetry points of Brillouin zone as reported in Ferrier et al. (2008), SL modes of the proposed elliptic PCs occur through the non-zero $k$-vector components in even A mode. The close inspections of regarding magnetic field profiles $\left(\mathrm{H}_{\mathrm{z}}\right)$ can be viewed from Fig. 1(b2) -for mode A- and Fig. 1(c2) - for mode B-. Note that the corresponding field distributions are calculated at a specific $k$-point signed with square markers in Fig. 1(a3). It can be inferred from the figures that the mode $A$ is even whereas B is an odd mode so that they don't couple to each other. Furthermore, the existing SL modes partially lie under the light-line. Although some portion of the SL band is above the light line, the PC slab could be sandwiched between either photonic bandgap claddings or total-internal reflection layers to avoid out-of-plane losses within the slow-light regime (Bushel et al. 2017).

The group index spectrum of the interested mode, even mode A, is plotted in Fig. 1(d). The calculated minimum group index for the even mode $\mathrm{A}$ is $n_{g}=63.56$. In the case of $10 \%$ group index variation, a bandwidth 


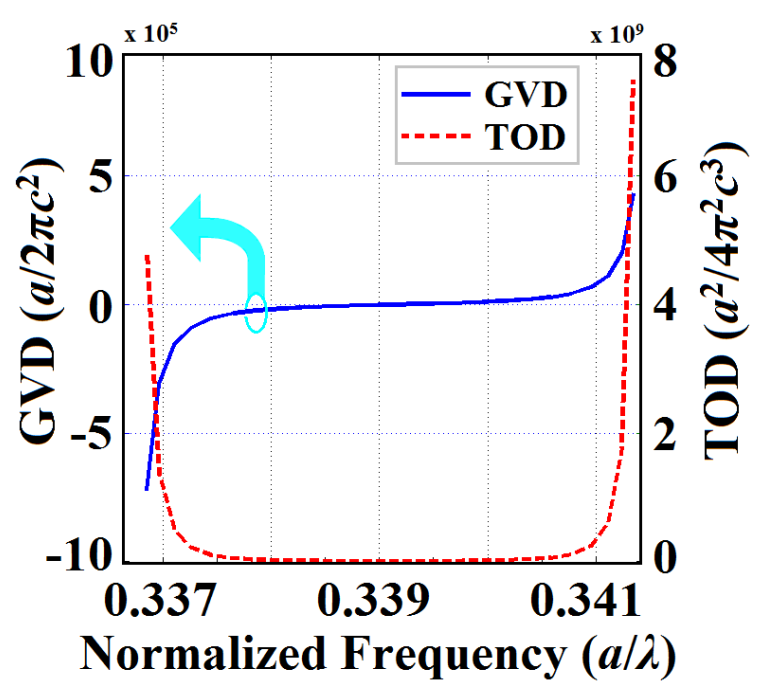

Figure 2. The calculated group velocity dispersion (GVD) and third-order dispersion (TOD) parameters for the even mode $\mathrm{A}$ as a function of operating frequencies.

that ranges from $a / \lambda=0.3379$ to $a / \lambda=0.3401$ is obtained. Thus, the resultant normalized bandwidth $(B W)$ becomes $B W=\Delta \omega / \omega_{c}=0.0065$, where $\omega_{c}$ is the radial frequency centered at a specified bandwidth. To operate at telecom wavelengths, the lattice constant is set as $a=525.45 \mathrm{~nm}$ so that the relative frequency bandwidth becomes $B W=1.26 \mathrm{THz}$ (or $10 \mathrm{~nm}$ around $1550 \mathrm{~nm}$ wavelength). Another essential parameter to exploit SL effect is Figure of Merit (FOM), which is defined by the product of the average group index and the normalized bandwidth, FOM $=\left\langle n_{g}\right\rangle B W$. In this case, the relative FOM equals $\mathrm{FOM}=0.4334$, which is reported to be FOM=0.554 in Üstün \& Kurt (2012) and comparable to the delay-bandwidth product of $\mathrm{FOM}=0.48$ reported in Elshahat et al. (2018). A recent study done by Ma et al. (2021) proposed a square lattice Si-based PCW configuration with extrinsic (InP) point-defects. The reported normalized delay bandwidth product in that paper is $\mathrm{FOM}=0.44$, which is still close to the reported value in our study. All above calculated parameters imply that the even mode A has a non-dispersive SL effect with a moderate bandwidth and FOM.

Other critical parameters to be determined in SL systems are the group velocity dispersion (GVD) and third-order dispersion (TOD). GVD is defined as $\mathrm{GVD}=\partial / \partial \omega\left(1 / v_{g}\right)=\partial^{2} k / \partial \omega^{2}$, indicating the group velocity dependence of slow-light on operating frequencies. GVD should be as small as possible in SL applications to reduce optical pulse distortion (Khurgin, 2005). TOD is another important parameter for SL devices implying the deformation on the envelope of propagating signal. TOD is defined by the derivative of GVD with respect to the angular frequency and it should be at minimum to reduce higher order dispersion effects (Engelen et al. 2006). Figure 2 represents the corresponding GVD and TOD graphs for degenerate even mode A, in which SL effect exists. Around the operating SL frequency of $a / \lambda=0.339$, the proposed system has zero GVD and TOD $=1.072 \times$ $10^{7}\left(a^{2} / 4 \pi^{2} c^{3}\right)$.

TE mode photonic band structure of 2D elliptic PC holes having the dimensions of $\left(a_{e}, b_{e}\right)=(0.40 a, 0.16 a)$ are traced along the edges of Brillouin zone, namely along X'$\Gamma-\mathrm{X}-\mathrm{M}-\Gamma$ path in order to observe the tendency of SL modes (see Fig. 3). The shaded regions along $\Gamma-X^{\prime}$ and $\Gamma$ $\mathrm{M}$ are inserted to determine the specified frequency interval where the SL effect is observed along $\Gamma-\mathrm{X}$ direction. It can be inferred from Fig. 3 that the degenerate modes of $\mathrm{A}$ and $\mathrm{B}$ along $\Gamma$-X tend to split towards other edges of Brillouin zone. Furthermore, available TE mode along $\Gamma$ - $X^{\prime}$ is mode $A$ whereas it is the odd mode along $\Gamma$-M in the SL frequency interval (shaded regions). Examining in detail the mode concerning mode profiles, an even mode cannot be excited along $\Gamma-\mathrm{M}$ direction since the existing mode in this SL interval is odd type. Considering the beam illumination along longitudinal $\Gamma-X$ direction, coupling of orthogonal modes along $\Gamma$-X' could be achieved only by the existence of cavities/resonator systems in the proposed SL structure (Suh et al. 2004). In our case, even though an even mode (mode $A$ ) exists along $\Gamma-X^{\prime}$, it cannot be excited via beam incidence along longitudinal $(\Gamma-\mathrm{X})$ direction since the PC structure does not include any disorders/defects. The calculated magnetic field profile along $\Gamma-X^{\prime}$ direction is given as an inset in Fig. 3. That situation brings to our mind that one can design a PC waveguide with finite thicknesses in order to obtain SL effect. That is because in the case of source incidence having even TE mode along $\Gamma$-X, no mode excitation occurs along $\Gamma$-M. That condition proves that strong SL excitation occurs only along longitudinal $x$-direction inside the defect-free PC waveguide with a finite thickness.

\section{SLOW-LIGHT EFFECT OF DESIGNED PHOTONIC CRYSTAL WAVEGUIDE INVESTIGATED BY 2D TIME-DOMAIN ANALYSES}

As mentioned previously, the PC waveguide having a finite thickness is formed by square lattice elliptical PC holes with the sizes of $\left(a_{e}, b_{e}\right)=(0.40 a, 0.16 a)$ in order to support a fundamental even TE mode. 2D time-domain analyses are carried out to calculate the coupling efficiency as well as to observe SL effect of designed defect-free PC waveguide. As schematically demonstrated in Fig. 4(a), the length of slow wave structure is fixed as $l=30 a$ but its width, $w_{2}$, is adjusted based on desired applications such as mode-order conversion and SL effect. A TE-polarized Gaussian pulse

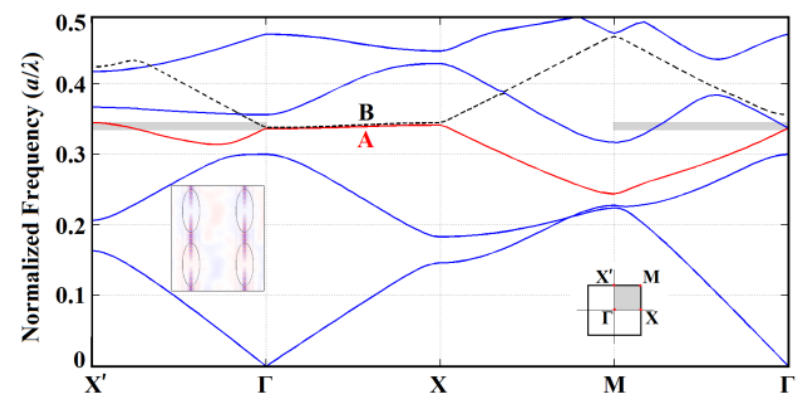

Figure 3. TE mode band structure of square-lattice elliptic PC holes with the dimensions of $\left(a_{e}\right.$, $\left.b_{e}\right)=(0.40 a, 0.16 a)$ along the edges of Brillouin zone, which is given as an inset in the right part of the figure. Shaded regions indicate the frequency interval in which SL effect is observed through $\Gamma$ - X direction. 
(a)

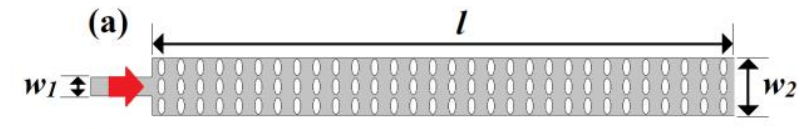

(b)

(c)

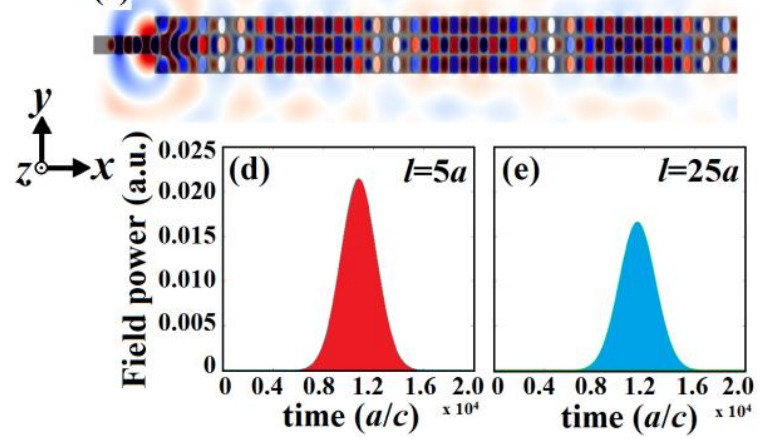

Figure 4. (a) Schematic view of 2D PC SL waveguide connected to an input dielectric waveguide with a width of $w_{1}=1 a$. Regarding steady-state field distributions when the sizes of SL waveguide are set to (b) $\left(l, w_{2}\right)=(30 a, 1 a)$ and (c) $\left(l, w_{2}\right)=(30 a, 3 a)$ when incident frequency is $a / \lambda=0.339$. Time delay graphs of SL modes detected at distances (d) $l=5 a$ and (e) $l=25 a$.

with a narrow bandwidth is injected from the input waveguide at a center frequency of $a / \lambda=0.339$, which has minimum GVD and TOD values. In this case, the slowed wave inside the SL structure becomes spatially localized, as can be noticed from Figs. 4(b) and 4(c). In these figures, only the input excitation port is shown and the waveguide is embedded into PMLs in order to suppress back reflections originating from the exit.

Remembering the magnetic field distribution of SL mode as in Fig. 1(b1), the SL mode, A, may also support higher order even modes. In this case, enlarging the width of SL waveguide enables a mode-order conversion of slowed light inside the system, as can be observed from Fig. 4(c). The coupling efficiency is calculated to be $41.4 \%$ for SL waveguide having a width of $w_{2}=1 a$ while this value decreases to $20.8 \%$ in the case of $w_{2}=3 a$. Comparing both efficiencies, it is clear that due to mode mismatch between the incident wave and propagating SL as well as the width mismatch occurring in between the input and the PC waveguides causes the regarding insertion losses. We should note that the mentioned coupling efficiencies are calculated without any coupler or anti-reflection coating mechanisms. The corresponding group indices are determined by using delay information for slowly-propagating wave in the designed configuration. Optical pulses detected in timedomain at the positions of $l=5 a$ and $l=25 a$ are presented in Figs. 4(d) and 4(e), respectively. The FDTD simulation is terminated after monitoring the signal. Apparently from time delay graphs, the SL mode retains its shape and the propagating pulse is not exposed to temporal broadening, which is the sign of low GVD and TOD. Comparing both Figs. 4(d) and 4(e), field intensity reduction is observed while propagating through the designed low-symmetric PC waveguide due to the light leakages occurring along the vertical $y$ - direction.
Nevertheless, propagation losses due to light leakages along the vertical direction can be suppressed by surrounding the PC waveguide with photonic bandgap materials (Kurt et al. 2011). The corresponding group index is calculated from the relation $n_{g}=c(\Delta t / \Delta l)$ and it is found to be $n_{g}=14.05$.

The group index of the proposed elliptical PC waveguide is also determined via 3D FDTD analysis in order to provide actual device modeling. Regarding lattice constant is kept as $a=520 \mathrm{~nm}$ in order to adjust the source incidence to operate at a wavelength of $1550 \mathrm{~nm}$. In this case, the designed dielectric PC slab possesses a thickness at around $220 \mathrm{~nm}$, which is a common value in SOI technology, and that SL waveguide having a width of $w_{2}=520 \mathrm{~nm}$ is laid upon the Silica $\left(\mathrm{SiO}_{2}\right)$ substrate. The cladding is set to be air. The calculated group index from delay information via 3D time-domain analysis is obtained to be $n_{g}=16.55$, which matches well with the calculated value from 2D FDTD analysis. The reason of group index reduction compared to the value of $n_{g}=63.56$ calculated from the band structure can be the fact that since we need to choose a finite width of PC waveguide in order not to excite higher-order modes, there appear an index mismatch at air-PC waveguide interfaces. Since the propagating field inside SL waveguide also penetrates into air in the direction transverse to propagation, corresponding group index as well as the power of localized fields diminishes.

Precise fabrication of 2D PCs with elliptical geometry is another issue to tackle. Realization of elliptical PC holes could be achieved with good quality (with minimized structural disorders) via two-beam interference lithography (Quiñónez et al. 2006) as well as laser holography techniques (Hung et al. 2010).

\section{CONCLUSION}

In conclusion, a defect-free low symmetric PC waveguide that enables SL effect is proposed. Due to the $\mathrm{C} 2$ rotational symmetry in the primitive cell, degenerate slow modes exist at higher TE bands. The even slow wave mode and its group index spectrum are investigated in frequency domain and the desired SL effect is numerically proved by FDTD analyses. The corresponding group index and $B W$ from the band structure are equal to $n_{g}=63.56$ and $B W=\Delta \omega / \omega_{c}=$ 0.0065 , which in turn gives a satisfactory delaybandwidth product value of $\mathrm{FOM}=0.4344$ in 2D PC structure. The proposed SL configuration also possesses a tolerable coupling efficiency around $41.4 \%$ without the need of any coupling mechanisms. In the proposed PC configuration, the existence of non-dispersive SL Bloch modes is achieved through the non-zero $k$-components of Brillouin zone, i.e. away from the band-edges. Moreover, changing the widths of proposed elliptic PC structure supports to excite higher order TE modes. Such a defectfree SL structure can be implemented for compact optical switching, optical delay lines and sensing applications.

\section{ACKNOWLEDGEMENT}

The author thanks to Prof. Kurt for fruitful discussions and advices about the study. 


\section{Conflicts of interest}

The author declares no conflicts of interest.

\section{REFERENCES}

Bagci F \& Akaoglu B (2015). Enhancement of buffer capability in slow light photonic crystal waveguides with extended lattice constants. Optical and Quantum Electronics, 47(3), 791-806.

Elshahat S, Khan K, Yadav A, Bibbò L \& Ouyang Z (2018). Slow-light transmission with high group index and large normalized delay bandwidth product through successive defect rods on intrinsic photonic crystal waveguide. Optics Communications, 418, 73-79.

Engelen R J P, Sugimoto Y, Watanabe Y, Korterik J P, Ikeda N, Van Hulst N F, ... \& Kuipers L (2006). The effect of higher-order dispersion on slow light propagation in photonic crystal waveguides. Optics express, 14(4), 1658-1672.

Ferrier L, Rojo-Romeo P, Drouard E, Letartre X, \& Viktorovitch P (2008). Slow Bloch mode confinement in 2D photonic crystals for surface operating devices. Optics express, 16(5), 3136-3145.

Giden I H \& Kurt H (2012). Modified annular photonic crystals for enhanced band gap properties and isofrequency contour engineering. Applied optics, 51(9), 1287-1296.

Giden I H, Turduev M \& Kurt H (2014). Reduced symmetry and analogy to chirality in periodic dielectric media. Journal of the European Optical Society-Rapid publications, 9.

Giden I H, Rezaei B \& Kurt H (2015). Method of implementing graded index media by symmetryreduced helical photonic structures. JOSA B, 32(10), 2153-2157.

Gumus M, Giden I H \& Kurt H (2018). Broadband selfcollimation in C2 symmetric photonic crystals. Optics letters, 43(11), 2555-2558.

Han X, Wang T, Tang J, Liu B, Wang B, He Y, \& Zhu Y (2015). Slow light with large group index-bandwidth product in ellipse-hole photonic crystal waveguides. Applied optics, 54(6), 1543-1547.

Hocini A, Maache M \& Khedrouche D (2018). Wideband and low dispersion slow light by altering the geometry of a photonic crystal waveguide. Optics Communications, 427, 396-404.

Hung Y J, Lee S L, Pan Y T, Thibeault B J, \& Coldren, L A (2010). Holographic realization of hexagonal two dimensional photonic crystal structures with elliptical geometry. Journal of Vacuum Science \& Technology B, Nanotechnology and Microelectronics: Materials, Processing, Measurement, and Phenomena, 28(5), 1030-1038.

Joannopoulos J D, Johnson S G, Winn J N, \& Meade R D (2008). Molding the flow of light. Princeton Univ. Press, Princeton, NJ. ISBN-10: 0691124566.

Johnson S G, \& Joannopoulos J D (2001). Block-iterative frequency-domain methods for Maxwell's equations in a planewave basis. Optics express, 8(3), 173-190.

Kassa-Baghdouche L \& Cassan E (2018). High efficiency slotted photonic crystal waveguides for the determination of gases using mid-infrared spectroscopy. Instrumentation Science \& Technology, 46(5), 534-54.

Kassa-Baghdouche L \& Cassan E (2019). Sensitivity analysis of ring-shaped slotted photonic crystal waveguides for mid-infrared refractive index sensing. Optical and Quantum Electronics, 51(10), 1-11.

Khodamohammadi A, Khoshsima H, Fallahi V \& Sahrai M (2015). Wideband slab photonic crystal waveguides for slow light using differential optofluidic infiltration. Applied optics, 54(5), 1002-1009.

Khurgin J B (2005). Optical buffers based on slow light in electromagnetically induced transparent media and coupled resonator structures: comparative analysis. JOSA B, 22(5), 1062-1074.

Khurgin J B \& Tucker R S (Eds.). (2018). Slow light: Science and applications. CRC press.

Krauss T F (2007). Slow light in photonic crystal waveguides. Journal of Physics D: Applied Physics, 40(9), 2666.

Kurt H, Giden, I H \& Ustun, K (2011). Highly efficient and broadband light transmission in $90^{\circ}$ nanophotonic wire waveguide bends. JOSA B, 28(3), 495-501.

Ma Y, Wu R \& Li L (2021). Research on slow light transmission with wide bandwidth and large normalized delay bandwidth product. Optoelectronics Letters, 17(7), 407-411.

Mirjalili S M \& Mirjalili S (2014). Oval-shaped-hole photonic crystal waveguide design by MoMIR framework. IEEE Photonics Technology Letters, 26(24), 2446-2449.

Mirjalili S M (2014). SoMIR framework for designing high-NDBP photonic crystal waveguides. Applied optics, 53(18), 3945-3953.

Moghaddam M K \& Fleury R (2019). Slow light engineering in resonant photonic crystal line-defect waveguides. Optics express, 27(18), 26229-26238.

Mori D \& Baba T (2005). Wideband and low dispersion slow light by chirped photonic crystal coupled waveguide. Optics express, 13(23), 9398-9408.

Oskooi A F, Roundy D, Ibanescu M, Bermel P, Joannopoulos J D \& Johnson S G (2010). MEEP: A flexible free-software package for electromagnetic simulations by the FDTD method. Computer Physics Communications, 181(3), 687-702.

Quiñónez F, Menezes J W, Cescato L, Rodriguez-Esquerre V F, Hernandez-Figueroa H \& Mansano R D (2006). Band gap of hexagonal 2D photonic crystals with elliptical holes recorded by interference lithography. Optics Express, 14(11), 4873-4879.

Schulz S A, Upham J, O'Faolain L \& Boyd R W (2017). Photonic crystal slow light waveguides in a kagome lattice. Optics letters, 42(16), 3243-3246.

Suh W, Wang Z \& Fan S (2004). Temporal coupled-mode theory and the presence of non-orthogonal modes in lossless multimode cavities. IEEE Journal of Quantum Electronics, 40(10), 1511-1518.

Üstün K \& Kurt H (2010). Ultra-slow light achievement in photonic crystals by merging coupled cavities with waveguides. Optics express, 18(20), 21155-21161.

Üstün K \& Kurt H (2012). Slow light structure with enhanced delay-bandwidth product. JOSA B, 29(9), 2403-2409. 
Trifonov T, Marsal L F, Rodriguez A, Pallares J \& Alcubilla $\mathrm{R}$ (2004). Effects of symmetry reduction in twodimensional square and triangular lattices. Physical Review B, 69(23), 235112.

Varmazyari V, Habibiyan H \& Ghafoorifard H (2014). Slow light in ellipse-hole photonic crystal line-defect waveguide with high normalized delay bandwidth product. JOSA B, 31(4), 771-779.

Wu H, Citrin D S, Jiang L Y \& Li X Y (2013). Polarizationindependent slow light in annular photonic crystals. Applied Physics Letters, 102(14), 141112.
Zhao Y, Zhang Y N \& Wang Q (2014). Slow-light optimization of polymer-infiltrated slot photonic crystal waveguide. IEEE Transactions on Nanotechnology, 13(4), 687-694.

Zhao Y, Zhang Y N, Wang Q \& Hu H (2015). Review on the optimization methods of slow light in photonic crystal waveguide. IEEE transactions on nanotechnology, 14(3), 407-426.

(C) Author(s) 2022. This work is distributed under https://creativecommons.org/licenses/by-sa/4.0/ 